\title{
Magnitude scales for electrocutaneous stimulation
}

\author{
PETER McCALLUM and HENRY GOLDBERG
}

\author{
The University of Melbourne \\ Department of Psychiatry, Austin Hospital, Heidelberg, Victoria, 3084, Australia
}

\begin{abstract}
The application of Stevens's power law to the sensation resulting from electrocutaneous stimulation is reviewed. Its use for data from individual observers as well as pooled data from several observers is discussed. Magnitude estimates were obtained from 33 observers of the sensation resulting from electrocutaneous stimulation over the median nerve. Seven mathematical functions were applied to the data and tested for goodness of fit. The power function with or without threshold correction factor did not emerge as better than alternative functions. Difficulties in using the power function in studies of individual differences are reviewed. It is concluded that there is no adequate reason at present to discard the linear function in favor of more complex functions in psychophysical scaling of sensation induced by electric shock.
\end{abstract}

The psychophysical power function was derived originally from extensive studies of the scaling of subjective loudness and brightness (Stevens, 1957). Application of magnitude estimation and other psychophysical scaling methods to a large number of perceptual continua followed. This process has been described as "an exciting game of testing the generality of the power-law principle" (Stevens, 1970). In the process, the power function assumed the status of a general law. Expressed mathematically: $R$ $=a \mathrm{Sb}$, where $\mathrm{R}$ is the median of magnitude estimates for each stimulus intensity, $\mathbf{S}$ the stimulus intensity, and " $a$ " and " $b$ " are constants. This is a power function with two constants and so is referred to hereafter as Equation $\mathrm{P}(2)$.

In 1958, Stevens, Carton, and Shickman reported a study designed to see if the sensation produced by electric shock applied to the fingers followed the general law. They reported that the relationship between current flow and magnitude estimates of apparent intensity were accurately described by a power function. However, they applied no statistical tests of goodness of fit and did not examine functions other than the power function. Furthermore, they presented the results of pooling of data from a number of observers.

Teghtsoonian (1973) asserts that there is now considerable evidence that the power law is not an artifact of pooling data over many observers but is evident in the behavior of individual observers. There is support for this from work in a number of perceptual continua (Marks \& J. C. Stevens, 1966; J. C. Stevens \& Guirao, 1964; Teghtsoonian \& Teghtsoonian, 1971). But the assertion is not universally supported. Pradhan and Hoffman (1963) reported a study of magnitude estimation of the

We are grateful to Dr. G. Stanley for help and advice. heaviness of lifted weights. They found that data from individual observers did not follow Stevens's law, although pooling of data from a number of observers did yield a power function. If the power function is to assume the status of a general law, it must be shown to apply consistently to data from individual subjects.

sternbach and Tursky (1964) presented a study of magnitude estimates of the sensation induced by shocks to the dorsum of the forearm. They reported that their results "confirm the existence of a power function for shock." However, again no tests of goodness of fit were applied, no alternative functions were tested, and their conclusions were based on data pooled from a number of observers.

Ekman, Frankenhaeuser, Levander, and Mellis (1964) reported a study of magnitude and category estimation of the sensation induced by shocks applied to the fingers. They also found that a power function yielded a good fit to the data. They included no statistical tests of goodness of fit, though it is clear from their paper that the power function gave a better fit than a linear function. But they also reported the results of pooling data from a number of observers and make no comments about the function best describing data from individual observers.

The power function in its original form has been amended (Ekman, 1958; Stevens, 1962) by subtraction from the stimulus intensity of "a constant related to threshold." Thus the relationship becomes a power function with three constants, $P(3)$.

$$
\mathrm{R}=\mathrm{A}(\mathrm{S}-\mathrm{b})^{\mathrm{c}} \ldots \ldots \text { Equation } \mathrm{P}(3)
$$

This equation has been widely used but not without reservations in some quarters. Poulton (1968) points out that an equation with three constants is likely to fit any set of psychophysical data fairly well. Even two constants give the curve-fitter considerable scope. He 
suggests that if Equation $P(3)$ is to have any special significance it must be shown to fit the data better than other functions with three constants. This matter has been little studied.

Rosner and Goff (1967) reported an attempt to fit Equation $\mathrm{P}(3)$ to data from magnitude estimation experiments using electric shock to the median nerve at the wrist. Unlike the previous studies, they utilized data from individual observers rather than pooled data. They had difficulty in fitting Equation $P(3)$ to some of their data. They concluded that a dual power function (which has five constants) better described the relationship between stimulus intensity and magnitude estimates. They postulated that this could be due to two separate populations of peripheral nerve fibres with different thresholds.

Beck and Rosner (1968) explored the problem further, using more sophisticated methods of curve-fitting and working with data from individual observers. They reported that a dual linear function (also with five constants) was as good as the dual power function. They investigated six functions altogether, and found that the two with five constants gave a significantly better fit than Equation $\mathrm{P}(3)$, which in turn gave a better fit than three functions with only two constants. They concluded from this that $P(3)$ emerged as the most parsimonious description of their data. However, they omitted the crucial step of testing other functions with three constants. Hence, a more skeptical conclusion from their paper is not that the power function gives the best fit, but simply that the more constants you include in the function, the better it fits.

Thus, it is yet to be established that a power function is the best description of the data from magnitude estimates from individual observers of the intensity of sensation produced by electric shock. Despite this, Stevens has recently reasserted that the power function is the most suitable (Stevens, 1970) and his assertion seems to have been accepted. Thus, Melzack (1973) cites the 1958 study of Stevens, Carton, and Shickman and states that "they found that the stimulus-sensation relationship is best described as a mathematical power function, a fact which has been confirmed by several other investigators." It seems from our review of the literature that Stevens's opinion has assumed the status of "fact" without sufficient justification.

So there seems to be a need for a study applying statistical tests of goodness of fit for several functions applied to data from magnitude estimates by individual observers using electrical stimulation. In particular, there is a need for a study of functions other than the power function which contain three constants. This paper reports such a study. This is of practical importance in the light of increasing interest in pain research using electrical stimulation (Higgins,
Tursky, \& Schwartz, 1971). This is essentially concerned with individual differences and does not permit the use of data pooled from a number of observers.

\section{METHOD}

Thirty-three normal young adults were studied, 13 male and 20 female. They were drawn from medical and paramedical personnel working in the hospital. All were naive to psychophysical experiments. Each was given a series of shocks via a pair of gold-plated electrodes, $8 \mathrm{~mm}$ in diam and containing electrode jelly, applied $5 \mathrm{~cm}$ apart over the median nerve at the wrist, with the anode proximal. The shocks used were $50-\mu \mathrm{sec}$ pulses from a Hewlett-Packard electromyograph with a voltage control modification for greater accuracy. A constant current stimulator is more commonly used for such experiments, but a constant voltage stimulator appears to be equally reliable (Schwartz, Emde, \& Shagass, 1965).

For each observer, the threshold was determined by the method of limits. Three descending and three ascending series of shocks were given.

Subsequently, each observer was given a series of shocks in a standardized randomized order at six intensities between 40 and $170 \mathrm{~V}$. This spans a range between just noticeable and somewhat uncomfortable for this pulse width. Five shocks were given at each intensity. No prescribed modulus was introduced. The range and order of the stimuli was standardied in view of the influence of these variables on the results (Poulton, 1968). Observers were asked to give a numerical estimate of intensity after each shock. Stimuli were given about $2 \mathrm{sec}$ after the observer had responded to the previous stimulus. No constraint was placed on the range of numbers used.

\section{RESULTS}

Medians for the magnitude estimates for each stimulus intensity were used for each observer. These were then fitted by the method of least squares to each of seven functions.

(1) $\mathrm{R}=\mathrm{aS}+\mathrm{b}$

Equation Lin (2)

a linear function with two constants, hence called Lin (2).

(2) $\mathrm{R}=\mathrm{a} \log \mathrm{S}+\mathrm{b}$

Equation $\log (2)$

(3) $\mathrm{R}=\mathrm{ASb}$

Equation $\mathrm{P}(2)$

(4) $\mathrm{R}=\mathrm{a}(\mathrm{S}-\mathrm{So})^{\mathrm{b}}$

Equation PT(2)

where So is the threshold for each observer as measured by the method of limits.

(5) $\quad \mathrm{R}=\mathrm{a}(\mathrm{S}-\mathrm{b})^{\mathrm{c}}$

Equation $\mathbf{P ( 3 )}$

(6) $\mathrm{R}=\mathrm{a} \log (\mathrm{S}-\mathrm{b})+\mathrm{c} \quad$ Equation $\log (3)$

(7) $\mathrm{R}=\mathrm{a} \mathrm{S}^{2}+\mathrm{bS}+\mathrm{c}$

Equation Par (3)

The last is a parabolic function with three constants and was used for no other reason than that it contains 
Table 1

Median Coefficient of Determination for 33 Subjects for Each of the Seven Functions Studied

\begin{tabular}{llc}
\hline Function & $\begin{array}{c}\text { Median } \\
\text { Coefficient of } \\
\text { Determination }\end{array}$ \\
\hline Lin & $(2)$ & .949 \\
Log & $(2)$ & .957 \\
P & $(2)$ & .946 \\
PT & $(2)$ & .949 \\
P & $(3)$ & .983 \\
Log & $(3)$ & .980 \\
Par & $(3)$ & .975 \\
\hline
\end{tabular}

Table 2

The Number of Times the Coefficient of Determination (to the Third Decimal Place) for the Function in the Column Exceeded that for the Function in the Row

\begin{tabular}{lrrrrrrr}
\hline & $\begin{array}{c}\text { Lin } \\
(2)\end{array}$ & $\begin{array}{c}\text { Log } \\
(2)\end{array}$ & $\begin{array}{c}\text { P } \\
(2)\end{array}$ & $\begin{array}{c}\text { PT } \\
(2)\end{array}$ & $\begin{array}{c}\text { P } \\
(3)\end{array}$ & $\begin{array}{l}\text { Log } \\
(3)\end{array}$ & $\begin{array}{l}\text { Par } \\
(3)\end{array}$ \\
\hline Lin (2) & & 18 & 20 & 19 & $32^{*}$ & $28^{*}$ & $30^{*}$ \\
Log (2) & 15 & & 14 & 18 & $31^{*}$ & $30^{*}$ & $30^{*}$ \\
P (2) & 13 & 19 & & 19 & $33^{*}$ & $28^{*}$ & $28^{*}$ \\
PT (2) & 14 & 15 & 14 & & $33^{*}$ & $27^{*}$ & $27^{*}$ \\
P (3) & 0 & 2 & 0 & 0 & & 11 & 16 \\
Log (3) & 4 & 0 & 5 & 6 & 22 & & 20 \\
Par (3) & 0 & 3 & 5 & 6 & 16 & 10 & \\
\hline
\end{tabular}

*Significantly different, $p<.01$.

three constants. The coefficients of determination were calculated for each function for data from each observer. The median values of the coefficients of determination for each function are shown in Table 1. Table 2 shows the number of times the coefficient of determination for the function in the column exceeded that for the function in the row.

These results were subjected to two-tailed binomial tests with Yates's correction for continuity (Siegel, 1956). The three functions with three constants were not significantly different. The four functions with two constants were also not significantly different. But all three functions with three constants were significantly superior to all four functions with only two constants $(\mathrm{p}<.01)$. Thus, in summary, $\mathrm{P}(3)=$ $\log (3)=\operatorname{Par}(3)>\operatorname{Lin}(2)=\log (2)=P(2)=$ PT(2).

As $P(3)$ has been regarded as the most suitable equation, the results obtained with this were studied further. One of the arguments advanced in favor of Equation $\mathrm{P}(3)$ is that the threshold correction factor [" $b$ " in Equation P(3)] emerges as closely related to the measured threshold. For our data, the median value of the threshold correction factor was $32 \mathrm{~V}$. This is close to the median value of the threshold as determined by the method of limits, which was $30 \mathrm{~V}$.

However, the individual values for the threshold correction factor varied widely, and in five instances it was less than zero.

Furthermore, there was a close correlation between the threshold correction factor and the value of the exponent [" $c$ " in Equation $\mathrm{P}(3)]$. Thus, as the value of the threshold correction factor became a negative number, the exponent tended to increase. When a linear regression line is fitted by the method of least squares to the two constants from each of the 33 observers, a correlation coefficient of .727 is obtained. This means that if the two are assumed to be linearly related, $52 \%$ of the variance in the value of the exponent is predicted from the value of the threshold correction factor.

\section{DISCUSSION}

These results allow several objections to be stated to use of Equation $\mathrm{P}(3)$ in magnitude estimation experiments with electric shock.

(1) It has not been shown to be superior to other functions with three constants. It is true that nonparametric tests of significance are of limited power. However, the methods used here proved powerful enough to demonstrate a highly significant difference between functions with three constants and functions with two constants. It is possible that a larger number of sets of observations or the use of a larger selection of stimulus intensities would show that $P(3)$ has a clear advantage. But with our data, no tendency emerged for $P(3)$ to be better, let alone a statistically significant tendency. Par (3) gave a better fit than $P(3)$ just as often as $P(3)$ gave a better fit than Par (3).

(2) It is questionable whether the value of the threshold correction factor has a meaningful relationship to threshold. Beck and Rosner (1968) report that the threshold correction factor "consistently exceeds absolute threshold by $10-20 \%$." However, this is based on the use of the medians of results from 14 observers. They showed that, for 14 observers, the median threshold correction factor fell steadily as pulse width was increased and remained $10 \%-20 \%$ above the threshold as determined by the method of limits. They cite this as buttressing the assertion of Stevens and Stevens (1963) that there is a close relationship between the correction factor and absolute threshold, although that paper reports a study of scaling of brightness.

These findings are interesting and require explanation. Our data appear consistent with this in that the median threshold correction factor was almost $10 \%$ above the absolute threshold as determined by the method of limits. But the wide scatter of individual values of the threshold correction must cast doubt on the use of median value for data from a number of subjects without further comment. Many experiments using magnitude estimation in the past have relied on pooling of data for a number of subjects. But in experiments designed to explore individual differences, this problem cannot be overlooked.

Ekman (1959) reports consistently finding a 
negative value of the threshold correction factor in work on the psychophysical scaling of brightness by fractionation. He speculates that this may be attributed to "a certain amount of sensory noise, resulting from spontaneous nervous activity." But this does not seem a very satisfactory explanation. It seems more appropriate to view it as a mathematical artifact devoid of physiological significance. If this is so, then the supposed relationship between " $b$ " in Equation P(3) and absolute threshold is dubious.

(3) Psychophysical experiments are primarily concerned with determining the slope of the relationship between stimulus and sensation $(S+R$ in our equations). For a power function, this is the exponent. The use of a threshold correction factor confounds the determination of the slope when Equation $\mathrm{P}(3)$ is used, or indeed any function which requires that $S$ be plotted on a nonlinear scale. This is no minor problem in that over half the variance in the value of the exponent for our observers is predicted from the value of the threshold correction factor.

These findings therefore do not support the view that Equation $\mathrm{P}(3)$ is the most suitable for use with data arising from psychophysical experiments with electric shock. Neither do they support the view that the original power function of Stevens's in either form $\mathrm{P}(2)$ or PT(2) is superior to other functions with two constants. It is interesting to note in this context that Beck and Rosner (1968) found that both Lin (2) and $\log (2)$ gave a significantly better fit $(p<.01)$ than $P(2)$. No such finding emerged with our data. But the data from both studies suggest that there is no adequate basis at this stage for discarding the linear function in favor of more complex equations, particularly in the light of the problem discussed in the previous paragraph. It can be argued that criteria other than goodness of fit should be considered in determining which function is appropriate. But the onus is then on the investigator to justify the criteria he uses.

To demonstrate the superiority of one function over another for psychophysical scaling, it is appropriate to use the widest possible range of stimulus intensities. We have endeavored to do this. However, the range that is available for electrocutaneous stimulation is extremely small, particularly in comparison to that available for studies of brightness or loudness. It may be that this limited range of stimulus intensities accounts for the failure of the power function to emerge as superior. But it does not seem unreasonable that electrical stimulation of the skin should be an exception to the power law. Such a stimulus is far removed from any naturally occurring stimulus. It bypasses the receptor organs in the skin which normally mediate between stimulus and nerve impulse, whether the stimulus is applied directly over a peripheral nerve or not (Rollman, 1969).

These results add weight to Poulton's comments about the need for rigorous statistical appraisal of functions used in psychophysical studies. Perhaps the power function has been applied too hastily to certain perceptual continua.

\section{REFERENCES}

Beck, C., \& Rosner, B. S. Magnitude scales and somatic evoked potentials to percutaneous electrical stimulation. Physiology and Behavior, 1968, 3, 947-953.

EKMAN, G. Two generalized ratio scaling method. Journal of Psychology, 1958, 45, 287-295.

EkMAN, G. Weber's law and related functions. Joumal of Psychology, 1959, 47, 343-352.

Ekman, G., Frankenhaeuser, M., Levander, S., \& Mellis, I. Scales of unpleasantness of electrical stimulation. Scandinavian Joumal of Psychology, 1964, 5, 257-261.

Higgins, J. D., Tursky, B., \& Schwhrtz, G. E. Shock-elicited pain and its reduction by concurrent tactile stimulation. Science, 1971, 172, 866-867.

Marks, L. E.. \& Stevens, J. C. Individual brightness functions. Perception \& Psychophysics, 1966, 1, 17-24.

Melzack, R. The puzzle of pain. Ringwood, Australia: Penguine, 1973.

Poulton, E. C. The new psychophysics: Six models of magnitude estimation. Psychological Bulletin, 1968, 69, 1-19.

Pradhan, P. L., \& Hoffman, P. J. Effect of spacing and range of stimuli on magnitude estimation judgements. Journal of Experimental Psychology, 1963, 66, 533-541.

Rollman, G. B. Electrocutaneous stimulation: Psychometric functions and temporal integration. Perception \& Psychophysics, 1969, 5, 289-293.

Rosner, B. S., \& GoFF, W. R. Electrical responses of the nervous system and subjective scales of intensity. In W. D. Neff (Ed.), Contributions to sensory physiology (Vol. 2). New York: Academic Press, 1967. Pp. 169-221.

Schwartz, M., Emde, J., \& Shagass, C. Comparison of constant current and constant voltage stimulators for scalp-recorded somatosensory responses. Electroencephalography and Clinical Neurophysiology, 1964, 17, 81-83.

SIEGEL, S. Nonparametric statistics for the behavioral sciences. New York: McGraw-Hill, 1956.

Sternbach, R. A., \& Tursky, B. On the psychophysical power function in electric shock. Psychonomic Science, 1964, 1, 217-218.

Stevens, J. C., \& Guirao, M. Individual loudness functions. Journal of the Acoustical Society of America, 1964, 36, 2210-2213.

Stevens, J. C., \& Stevens, S. S. The brightness function: Effects of adaptation. Journal of the Optical Society of America, 1963, 53, 375-385.

Stevens, S. S. On the psychophysical law. Psychological Review, 1957, 64, 153-181.

STEVENS, S. S. The surprising simplicity of sensory metrics. American Psychologist, 1962, 17, 29-39.

StEvens, S. S. Neural events and the psychophysicsl law. Science, 1970, 170, 1043-1050.

Stevens, S. S., Carton, A. S., \& Shickman, G. M. A scale of apparent intensity of electric shock. Journal of Experimental Psychology, 1958, 56, 328-334.

Teghtsoonian, M., \& Teghtsoonian, R. How repeatable are Stevens's power law exponents for individual subjects? Perception \& Psychophysics, 1971, 10, 147-149.

Teghtsoonian, R. Range effects in psychophysical scaling and a revision of Stevens's law. American Journal of Psychology, 1973, 86, 3-27.

(Received for publication June 3, 1974; revision received July 31,1974 .) 